\title{
Alimentación, estados afectivos y actividad física en estudiantes universitarios mexicanos durante la pandemia por COVID-19
}

\section{Nutrition, affective states and physical activity in Mexican university students during the COVID-19 pandemic}

\author{
Mayra Lizeth Salgado-Espinosaa , Luis Armando Cepeda-Gaytana \\ a Centro de Investigación, Desarrollo e Innovación Vizcaya, Universidad Vizcaya de las Américas campus Saltillo, México
}

\section{Resumen}

Introducción: La pandemia por la COVID-19 ha provocado un importante cambio en la cotidianidad del alumnado universitario impactando en su salud a través de sus hábitos y estados emocionales. Objetivo: Analizar los hábitos de alimentación e identificar su relación con los estados emocionales, así como los hábitos asociados a la práctica de actividad física en estudiantes universitarios de la Universidad Vizcaya de las Américas de la ciudad mexicana de Saltillo (Coahuila) durante la pandemia por la COVID-19. Metodología: Diseño cuantitativo, correlacional y transversal. Se realizó un muestreo no probabilístico por conveniencia. Participaron 758 estudiantes universitarios, con una media de edad de 22.42 años (D.E.= 6.09). Resultados: Se encontró que el $54.7 \%$ incrementó su práctica de actividad física y el $45.7 \%$ reportó comer más que antes de la pandemia. El $31.3 \%$ presentó un nivel de "comedor no emocional", mientras que el 25.5\% alcanzó un puntaje de "comedor emocional". Se encontró una correlación positiva significativa ( $r h o=0.497, p<0.05$ ) entre el índice de comedor emocional y estados afectivos negativos. El estado afectivo experimentado con mayor frecuencia fue estar atento/a ( $\bar{x}=2.92$, D.E.=1.13). Conclusiones: El estudio de las implicaciones de la pandemia en los hábitos de salud y estados emocionales de los universitarios permitirá generar estrategias para la toma de decisiones oportunas para la adopción y mantenimiento de hábitos saludables.

Palabras clave: Actividad física; alimentación; emociones; universitarios; México, COVID-19.

\begin{abstract}
Introduction: The COVID-19 pandemic has caused an important change in the daily life of university students, impacting their health through their habits and emotional states. Objective: To analyze eating habits and identify their relationship with emotional states, as well as habits associated with the practice of physical activity in university students of the Universidad Vizcaya de las Américas in the Mexican city of Saltillo (Coahuila) during the COVID-19 pandemic. Methodology: Quantitative, correlational and cross-sectional design. A non-probabilistic convenience sampling was carried out, 758 university students participated, with a mean age of 22.42 years (SD $=6.09$ ). Results: It was found that $54.7 \%$ increased their physical activity practice and $45.7 \%$ reported eating more than before the pandemic. 31.3\% presented a level of "non-emotional eater", while $25.5 \%$ reached a score of "emotional eater". A significant positive correlation ( $r$ o $=0.497, p<0.05$ ) was found between the emotional eater index and negative affective states. The most frequently experienced affective state was being attentive $(\bar{X}=2.92$, S.D. = 1.13). Conclusions: The study of the implications of the pandemic in the health habits and emotional states of university students will allow generating strategies for making timely decisions for the adoption and maintenance of healthy habits.
\end{abstract}

Keywords: Physical activity; nutrition; emotions, university students; Mexico; COVID-19. 


\section{Introducción}

- a pandemia generada por la enfermedad por coronavirus (COVID-19) ha tenido un gran impacto en todas las esferas de la vida de las personas debido a la amenaza al estado general de salud que ha implicado un gran reto para los gobiernos y sistemas de salud a nivel mundial.

Desde que se identificó el virus, en diciembre del 2019 en Wuhan (China), se han presentado grandes cambios en los hábitos de vida de toda la población. Esto es debido a las diversas estrategias y medidas sanitarias que debieron ser implementadas de forma obligatoria para evitar la propagación del virus, entre las cuales destacan: el uso de mascarillas o cubrebocas, el distanciamiento social y el confinamiento obligatorio. Dichas medidas fueron implementadas en México desde el 24 de marzo de 2020, declarándose seis días después como una "emergencia sanitaria por causa de fuerza mayor" (Aburto-Morales et al., 2020), que ha traído afectaciones a nivel físico, psicológico y social (RuizZaldibar et al., 2021).

La población universitaria no ha sido la excepción. Tal como plantean Salgado-Espinosa y Álvarez (2020), este grupo se encuentra condicionado por su contexto y sus propias experiencias, agravado por el impacto de la pandemia sobre los sistemas de salud, económicos y sociales. A esto se suma la incertidumbre del alumnado (Johnson, Saletti-Cuesta, \& Tumas, 2020) respecto a su proceso formativo y sus actividades cotidianas restringidas por la situación de la pandemia.

Tal como sugieren Giuntella, Hyde, Saccardo y Sadoff (2021), la población adolescente y adulta joven se ha convertido en un grupos etarios de especial preocupación debido a los grandes cambios en su educación y situación de vida, pudiendo además sufrir las afectaciones económicas que trajo consigo la pandemia. Igualmente, la dinámica cotidiana de los universitarios cambió totalmente al optar por un modelo de educación en línea, limitando las interacciones con sus compañeros, lo que también ha traído como consecuencia la disminución de la motivación y el empeoramiento de sus comportamientos de salud (Gonzalez-Ramirez, Mulqueen, Silverstein, Mulqueen, \& BuShell, 2020).

En la investigación realizada por Ruiz-Zaldibar et al. (2021) en universitarios españoles, encontraron que los estilos o hábitos de vida saludable de los universitarios empeoraron durante el confinamiento. Además, estos investigadores distinguieron las diferencias de acuerdo al sexo, en el que las mujeres se vieron mayormente afectadas en el plano psicológico, a través de los aspectos relacionados a las relaciones sociales y familiares, así como en sentimientos o pensamientos positivos; mientras que en los hombres disminuyó la práctica de actividad física.

Otro hábito de gran impacto para la salud es la alimentación. Una adecuada alimentación contribuye a garantizar un estado de salud óptimo y evitar que se presente un déficit que se traduzca en un problema de salud. Sin embargo, de acuerdo con Reyes y Canto (2020), los hábitos de alimentación de los universitarios se ve influido por modas dietéticas, la falta de tiempo libre, el estrés, las actividades laborales, el poco conocimiento de la preparación de alimentos o el escaso dinero disponible. Los hábitos alimentarios se convierten en un comportamiento consciente, repetitivo, pero sobre todo colectivo, ya que se construye a partir de influencias sociales y culturales (Varela, 2016).

Chowdhury (2020) sostiene que la alimentación juega un papel fundamental en nuestro organismo en la prevención y respuesta inmune contra la COVID-19. De tal forma, una nutrición adecuada se puede convertir en un factor de protección (Ruiz-López, Felipe-Ortega-Fonseca, Vázquez-Curiel, \& Balderrama-Carmona, 2021).

Barrera et al. (2008), en su estudio con universitarios, resalta que los estudiantes se encuentran en un período crítico que juega un papel crucial en su salud y su pronóstico puede mejorar en la medida que seleccionen y preparen los alimentos. La forma de elaborar los alimentos es importante ya que involucra la manera en la que se conservan, modifican o pierden las cualidades, la manera en que se manipulan, así como la forma en la que se almacenan y se cocinan (Sedó, Chinnock, \& Piedra, 2000).

Por lo que a la alimentación respecta, el conocimiento adquirido, y las actividades relacionadas con la preparación de los alimentos, son un momento decisivo para la adquisición de un estilo de vida saludable (Pérez, Vega-García, \& Romero-Juárez, 2007). Sin embargo, en el estudio de Sainz, Ferrer y Sánchez (2016) con universitarios de la ciudad española de Barcelona se encontró que la percepción de capacidades culinarias era muy baja y la mayoría consumía alimentos procesados.

Por otro lado, el aspecto psicológico se ha convertido en un tema importante al estudiar los hábitos de salud, ya que es importante identificar que el conocimiento en sí mismo no es un determinante para llevar a cabo un comportamiento. Al respecto, en un estudio de Peña-y-Lillo (2019) se encontró que las actitudes, las percepciones normativas y el control se asociaban con 
la intención para optar por una alimentación saludable, en el que intervienen las creencias respecto a este tipo de conductas.

Dentro de los principales motivos por los que los universitarios no cuidan su alimentación, aún desde antes de la situación de pandemia, se encuentra la preferencia por la comida basura (Salgado-Espinosa \& Álvarez, 2020). Desde el punto de vista nutricional, Fajardo, Camargo, Buitrago, Peña y Rodríguez (2016) reporta que es importante el tener presente las características organolépticas de los alimentos a la hora de la preparación, para promover la elección de alimentos saludables con mayor calidad nutricional, que las personas sientan agrado por las comidas y que no se convierta en una mera elección de alimentos.

La calidad de los alimentos es una de las cualidades que se deben apreciar en los alimentos. Se puede abordar desde diferentes perspectivas con la finalidad de satisfacer las necesidades alimentarias de los individuos, considerando que los atributos que hacen la diferencia son: la preparación de los alimentos con una adecuada inocuidad, las cualidades sensoriales, la calidad, el valor nutricional y la forma de presentación del mismo (Noguera et al., 2018). La correcta elaboración de los alimentos tienen como objetivo que sean agradables sin perder sus propiedades, que se aprovechen de manera eficaz y que preserven las propiedades y el valor nutricional (Achón, González, \& Varela 2018; López et al., 2021).

Se han realizado investigaciones sobre los cambios en los estilos de vida durante el confinamiento (ÁlvarezBermudez \& Meza-Peña, 2021; Ammar et al., 2020; Osorio \& Prado, 2021; Romero-Blanco et al., 2020). En estos estudios se asoció el confinamiento con la disminución de la práctica de actividad física, el incremento de tiempo dedicado a actividades sedentarias, así como la adopción y el mantenimiento de un patrón no saludable de consumo de alimentos como la falta de control y la ingesta de snacks entre comidas, atribuyendo dichos cambios a estados emocionales negativos asociados al aburrimiento y la ansiedad.

Los cambios aplicados en la conducta alimentaria crean una barrera para hacer frente a las emociones, afectando más a los individuos que experimentan diversas emociones negativas que los deja en una situación de vulnerabilidad, incrementando la probabilidad de consumir más alimentos de lo habitual. Este perfil se ha definido como comedor emocional (Tazeoğlu, Bozdoğan, \& İdiz, 2021). En un estudio realizado por Peña y Reidl (2015) menciona que los comedores emocionales incrementan su consumo de alimentos cuando se presentan cuadros de situaciones desapacibles. Sin embargo, en los comedores no emocionales no se observan modificaciones de acuerdo al patrón alimentario referente a las emociones que siente. Las emociones tienen un efecto poderoso sobre las personas, basándose principalmente en la selección de los alimentos y en la forma en que se van desarrollando los hábitos alimentarios (Sánchez Benito \& Pontes Torrado, 2012).

Las variaciones de los comedores emocionales dan respuesta a las emociones representativamente negativas, lo cual se presenta un factor de riesgo referente a aliviar sentimientos asociados al estrés, el miedo, la frustración, etc., sin considerar las señales de hambre y saciedad, para escapar de dichos sentimientos (Osorio \& Prado, 2021; Rojas \& García-Méndez, 2017).

Asimismo, la relación entre alimentación, actividad física y estados emocionales ha sido demostrada a través de numerosos estudios (Celorio-Sardà et al., 2021; Giuntella et al., 2021; Son, Hegde, Smith, Wang, \& Sasangohar, 2020) que indican que dichos hábitos son elementos clave en el bienestar y el éxito académico, donde incluso la baja actividad física es considerada un factor de riesgo para la depresión durante la pandemia.

Los hallazgos anteriores hacen necesario que se profundice en los estados afectivos. Según Watson y Tellegen (1985, citado por: Moral, 2011) los estados afectivos se dividen en dos dimensiones: el afecto positivo y el negativo. El afecto positivo se asocia a la motivación, el éxito o la afiliación o apego hacia otras personas; mientras que el afecto negativo se refiere a sentimientos desagradables, de miedo, inseguridad o fracaso.

Los estados emocionales que se han presentado durante la pandemia han derivado del cambio abrupto en la dinámica cotidiana (Johnson et al., 2020), en lo cual ha tenido una importante influencia la cantidad de información difundida a través de medios digitales, que incluye información falsa y fake news influyendo en el razonamiento (Catalán-Matamoros, 2020). Esta situación ha constituido un importante punto de partida para la implementación y acceso a programas que favorecen la salud mental de la población.

Por lo tanto, los hábitos de vida saludable y los estados emocionales tienen una relación importante que se ha visibilizado con la pandemia, en el que los comportamientos alimentarios se ven envueltos en la regulación del estado emocional a través del tipo de alimentos ingeridos (Amatori et al., 2020). La práctica de actividad física se asocia a la autorregulación, la autoeficacia y el estado de ánimo, que son variables psicológicas relacionadas con el comportamiento alimentario (Annesi, 2012; Salgado-Espinosa \& Álvarez, 2020). 
La importancia del estudio de los hábitos de vida saludable y su relación con los estados emocionales radica en que la pandemia ha suscitado importantes factores de riesgo para la adopción y mantenimiento de hábitos de vida no saludables. Esto ha sido debido al incremento en de la preocupación por la propia salud y de sus seres queridos, la dificultad para concentrarse en las clases virtuales, la modificación de los patrones de sueño, el aislamiento social, los pensamientos depresivos e y la ideación suicida (Son et al., 2020).

El objetivo de este trabajo es analizar los hábitos de alimentación e identificar su relación con los estados emocionales, así como los hábitos asociados a la práctica de actividad física en estudiantes universitarios de la Universidad Vizcaya de las Américas de la ciudad mexicana de Saltillo (Coahuila), durante la pandemia por la COVID-19.

\section{Métodos}

\section{Diseño}

Investigación cuantitativa, descriptiva, con alcance correlacional, de corte transversal.

\section{Participantes}

Se realizó un muestreo no probabilístico por conveniencia, con una muestra de 758 estudiantes de Universidad Vizcaya de las Américas de la ciudad mexicana de Saltillo. El $71.1 \%$ eran mujeres, el $28.5 \%$ hombres y el $0.4 \%$ prefirió no precisar su sexo. La media de edad fue de 22.42 años (D.E.=6.09). El 80.8\% de la muestra indicó ser soltero/a, el $9.6 \%$ casado/a, el $7.4 \%$ en unión libre, el $1.8 \%$ divorciado/a y el $0.3 \%$ viudo/a.

Respecto a los estudios, el $29.6 \%$ estaba cursando una titulación de Ciencias de la Salud (Psicología o Nutrición); el 27.3\% una titulación de Administración (Administración de Empresas, Contaduría Pública 0 Comercio Internacional); el $18.5 \%$ una titulación de Ciencias Sociales (Derecho, Criminología o Educación); el 15.2\% la titulación de Gastronomía; el 6.4\% del área de Creativas (Ciencias y técnicas de la comunicación, Diseño Gráfico o Arquitectura) y el 3.2\% la titulación de Ingeniería.

Al finalizar la recogida de muestra el $49 \%$ se encontraba estudiando el primer cuatrimestre (primer curso académico), el $27.6 \%$ el cuarto cuatrimestre (segundo curso académico) y el $23.4 \%$ el séptimo cuatrimestre (cuarto curso académico).

Los criterios de inclusión para la participación en el estudio fueron los siguientes: pertenecer a la Universidad
Vizcaya de las Américas campus Saltillo (Coahuila) y estar matriculado en el momento de la recogida de datos.

\section{Instrumentos}

Se recopilaron datos sociodemográficos de edad, sexo, titulación y semestre, así como datos antropométricos de peso y talla, para el cálculo del IMC de acuerdo a la fórmula de Quetelet (IMC=Peso/Talla2). Asimismo, se realizaron seis preguntas cerradas referentes a los hábitos de alimentación, preparación de alimentos, causas por las que no se preocupan por su alimentación y cambios a raíz de la pandemia por la Covid-19.

Se aplicó el Cuestionario del Comedor Emocional de Garaulet et al. (2012), que consta de 10 ítems que evalúan la relación de la ingesta de alimentos y las emociones, permitiendo detectar personas con mayor impulsividad frente a la alimentación (González, 2018). Algunos ejemplos de preguntas de este cuestionario son: "¿Tienes antojos por ciertos alimentos específicos?" - "¿Tienes problemas para controlar las cantidades que comes de ciertos alimentos?". Las opciones de respuesta son "nunca" (0 puntos), "a veces" (1 punto), "generalmente" (2 puntos) o "siempre" (3 puntos). Al sumar las puntuaciones del cuestionario, se puede determinar si el participante es "comedor no emocional" (0 a 5 puntos), "comedor poco emocional" (6 a 10 puntos); "comedor emocional" (11 a 20 puntos); o "comedor muy emocional" (21 a 30 puntos). Además, la escala se divide en tres categorías: 1) desinhibición, relacionado con la falta de control en el consumo de alimentos (ítems 4, 5, 6, $8,9,10) ; 2$ ) tipo de alimento, refiriendo al tipo de alimento consumido de acuerdo al estado emocional o situación (ítems 2 y 3); y culpa, relacionado con la báscula o el consumo de alimentos prohibidos (ítems 1 y 7 ) (Romero, 2018). La confiabilidad del instrumento fue de 0.84 .

Para evaluar el estado emocional, se aplicó la Escala de Afecto Positivo y Negativo PANAS-20 de Watson, Clark y Tellegen (1988), en su versión validada al español de Robles y Páez (2003). El instrumento consta de 20 palabras que representan diferentes emociones, dividiéndose en 10 emociones referentes al afecto positivo, tales como "Estimulado/a", "Inspirado/a" u "Orgulloso/a", entre otras (ítems 1, 3, 5, 9, 10, 12, 14, 16, 17 y 19); y otras 10 emociones de afecto negativo, como "Tenso/a", "Disgustado/a" u "Hostil", entre otras (ítems 2, 4, 6, 7, 8, $11,13,15,18$ y 20). En las 20 palabras-emociones de la escala en el que el participante debe valorar el grado en el que las experimenta ("Nada", "Un poco", "Bastante", "Mucho", "Muchísimo"). Este instrumento ha sido utilizado en la investigación de Rojas-Vichique et al. (2020) para 
correlacionar las emociones positivas y negativas con el comportamiento alimentario. La confiabilidad del instrumento en la presente investigación fue de 0.85 .

También se aplicó el Cuestionario Mundial sobre Actividad Física (Global Physical Activity Questionnaire, [GPAQ]) en su versión reducida, creado por la Organización Mundial de la Salud (OMS, 2014). Este instrumento recaba información sobre el tiempo empleado al caminar, en actividades con una intensidad moderado y vigorosa, así como en actividades sedentarias (Mantilla \& GómezConesa, 2007). El GPAQ ha sido ampliamente validado y cuenta con altos niveles de confiabilidad, por lo que la OMS recomienda su uso (Bravo-Cucci et al., 2020).

\section{Procedimiento}

Se solicitó la autorización por parte del director general del campus universitario de Saltillo. Debido a la situación de pandemia, se optó por la aplicación de instrumentos a través de la herramienta Google Forms ${ }^{\circledR}$, que fue compartido a través de redes sociales a todos los grupos y titulaciones de la Universidad Vizcaya de las Américas campus Saltillo, Coahuila.

Dentro de las consideraciones éticas, antes de iniciar el instrumento se hizo mención del carácter confidencial y la participación voluntaria en la investigación, añadiendo también el objetivo de la investigación y un correo electrónico de la responsable del proyecto.

\section{Análisis estadístico}

El análisis de datos se realizó mediante el Paquete Estadístico para Ciencias Sociales (Statistical Package for Social Sciences [SPSS]) versión 23. Se realizaron análisis descriptivos de frecuencias para analizar las características sociodemográficas, hábitos de alimentación, y actividad física. Se realizó el cálculo de la media y la desviación estándar de los ítems del Cuestionario del Comedor Emocional y en la Escala de Estados Afectivos.

Se realizaron análisis de la U de Mann-Whitney para comparar el Índice de Comedor Emocional entre sexos; así como la prueba de Kruskall-Wallis, entre quienes consideran que comen más que antes de la pandemia, los que comen menos que antes y lo que no cambiaron sus hábitos de alimentación.

También, se analizó la relación entre el índice de Comedor Emocional, estados afectivos positivos y estados afectivos negativos, a través de los análisis de correlación de Spearman.

\section{Resultados}

\section{Alimentación}

En relación a los hábitos alimentarios de los estudiantes durante el confinamiento, el $45.7 \%$ refirió que come más que antes; el $20.3 \%$, un poco menos que antes y el $33.9 \%$ ha expresado que no ha presentado cambios.

Es importante señalar que un $90.6 \%$ de la población estudiantil reportó que sí le preocupaba el cuidar su alimentación; mientras que a un $9.4 \%$ no le preocupaba este aspecto. De estos últimos, las razones por las que no les preocupó fueron porque no veían ninguna consecuencia $(20.7 \%)$; la preferencia hacia la comida basura (18\%); el no tener el conocimiento de cómo cuidar su alimentación (18\%); que casi no les daba hambre (11.3\%); la falta de tiempo (10.7\%); porque su madre 0 alguien más eran los encargados del cuidado de su alimentación (9.3\%); que están muy cansados (7.3\%); y/o por falta de recursos económicos (4.7\%).

El promedio de comida que se preparaban los/las estudiantes al día fue de $56.53 \%$ (D.E. 30.06). Mientras que para el $59 \%$ de los universitarios, la persona encargada de la mayor parte de la preparación de los alimentos que consumían era su madre; en segundo lugar, fue el universitario mismo (32.1\%); y en tercer lugar la pareja $(3.4 \%)$.

La principal forma de preparación de alimentos para el $41.9 \%$ de los universitarios fue guisado; el $21.2 \%$ hervido; el $14.8 \%$ asado; el $13.9 \%$ frito; y el $8.1 \%$ horneado.

\section{Comedores emocionales}

Para identificar la relación entre los estados emocionales y la alimentación, se aplicó el Cuestionario del Comedor Emocional de Garaulet et al. (2012). En la Tabla 1 se muestra que la situación que se presentó con mayor frecuencia fue la de tener antojos por ciertos alimentos específicos con una media de 2.39 (D.E. .74) y, en segundo lugar, se encontró que los universitarios comían cuando estaban estresados, enfadados o aburridos con una media de 2.12 (D.E. 0.93).

En cuanto a los niveles identificados, el $38.1 \%$ se ubicó en un nivel de "comedor no emocional"; el $31.3 \%$ en "comedor poco emocional"; el $25.5 \%$ en "comedor emocional"; y el $5.1 \%$ en "comedor muy emocional".

En la Tabla 2 se presentan los análisis estadísticos comparativos entre el índice de Comedor Emocional, sexo y cambios en la alimentación durante la situación de 
pandemia. En torno al Índice de Comedor Emocional y la diferencia entre sexos, se realizaron análisis comparativos de la $U$ de Mann Whitney encontrando diferencias significativas. Las mujeres obtuvieron un rango promedio más alto (394.28) que los hombres (335.69).

Se realizó la prueba de Kruskall-Wallis, encontrando diferencias significativas $(p<0.05)$ entre quienes consideran que comen más que antes de la pandemia, los que comen menos que antes y los que no cambiaron sus hábitos de alimentación, siendo más alto el Índice de Comedor emocional en quienes comen más que antes de la pandemia.

Tabla 1. Media y Desviación Estándar de la Escala del Comedor Emocional de Garaulet et al. (2012).

\begin{tabular}{|l|c|c|}
\hline & $\overline{\mathrm{X}}$ & D.E. \\
\hline $\begin{array}{l}\text { ¿La báscula tiene un gran poder } \\
\text { sobre ti? ¿Es capaz de cambiar tu } \\
\text { estado de humor? }\end{array}$ & 1.88 & 0.88 \\
\hline $\begin{array}{l}\text { ¿Tienes antojos por ciertos } \\
\text { alimentos especificos? }\end{array}$ & 2.39 & 0.74 \\
\hline $\begin{array}{l}\text { ¿Te cuesta parar de comer alimentos } \\
\text { dulces, especialmente chocolate? }\end{array}$ & 1.63 & 0.81 \\
\hline $\begin{array}{l}\text { ¿Tienes problemas para controlar } \\
\text { las cantidades de ciertos alimentos? }\end{array}$ & 1.76 & 0.81 \\
\hline $\begin{array}{l}\text { ¿Comes cuando estás estresado, } \\
\text { enfadado o aburrido? }\end{array}$ & 2.12 & 0.93 \\
\hline $\begin{array}{l}\text { ¿Comes más de tus alimentos } \\
\text { favoritos, y con más descontrol, } \\
\text { cuando estás solo }\end{array}$ & 1.92 & 0.88 \\
\hline $\begin{array}{l}\text { ¿Te sientes culpable cuando tomas } \\
\text { alimentos "prohibidos", es decir, } \\
\text { aquellos que crees que no deberías, } \\
\text { como los dulces o snacks? }\end{array}$ & 1.98 & 1.04 \\
\hline $\begin{array}{l}\text { Por la noche, cuando llegas } \\
\text { cansado de trabajar, ¿es cuando } \\
\text { más descontrol sientes en tu } \\
\text { alimentación? }\end{array}$ & & \\
\hline $\begin{array}{l}\text { Estás a dieta, y por alguna razón } \\
\text { comes más de la cuenta, entonces } \\
\text { piensas que no vale la pena y } \\
\text { ¿comes de forma descontrolada } \\
\text { aquellos alimentos que piensas que } \\
\text { más te van a engordar? }\end{array}$ & 1.66 & 0.85 \\
\hline $\begin{array}{l}\text { ¿Cuántas veces sientes que la } \\
\text { comida te controla a ti en vez de tú } \\
\text { a ella? }\end{array}$ & 1.45 & 0.82 \\
\hline
\end{tabular}

Nota: Se evaluó mediante una escala de "Nunca” "A veces" "Generalmente" "siempre". 
Tabla 2. Análisis comparativo de la U de Mann-Whitney entre Índice de Comedor Emocional y sexo.

\begin{tabular}{|l|c|c|c|c|c|}
\hline & Sexo & $\mathbf{N}$ & $\begin{array}{c}\text { Rango } \\
\text { Promedio }\end{array}$ & Estadístico & Sig. \\
\hline \begin{tabular}{l} 
Índice de $\begin{array}{l}\text { Comedor } \\
\text { Emocional }\end{array}$ \\
\cline { 2 - 5 }
\end{tabular} & Hombre & 216 & 335.69 & $\mathrm{U}=$ & 0.001 \\
\cline { 2 - 4 } & $\begin{array}{c}\text { Mujer } \\
\text { Como más que } \\
\text { antes }\end{array}$ & 538 & 394.28 & 49074.00 & \\
\cline { 2 - 4 } & $\begin{array}{c}\text { Como menos } \\
\text { que antes }\end{array}$ & 154 & 374.61 & \multirow{2}{*}{60.033} & \\
\cline { 2 - 5 } & $\begin{array}{c}\text { No cambió mi } \\
\text { alimentación }\end{array}$ & 257 & 300.30 & & \\
\hline
\end{tabular}

\section{Estados emocionales}

En la Tabla 3 se observa que, respecto a los estados afectivos positivos, la media más alta refirió a sentirse atento/a con un 2.92 (D.E. 1.13), y la media más baja fue en sentirse estimulado/a con 2.19 (D.E. .963). Mientras que, en torno a los estados afectivos negativos, la media más alta se encontró en sentirse tensola con 2.37 (D.E. 1.05) y la más baja se reportó en sentirse hostil con 1.63 (D.E. .82).

Al realizar los análisis de correlación de Spearman entre estados afectivos (positivos y negativos) y el índice de Comedores emocionales se encontró una correlación estadísticamente significativa, positiva y moderada (rho=0.497, $p<0.05$ ) entre el afecto negativo y el índice de comedores emocionales; mientras que se encontró una baja correlación significativa negativa (rho=-0.116, $p<0.05)$ entre éste último y el afecto positivo. Asimismo, se encontró una baja correlación positiva estadísticamente significativa ( $r h o=0.171, p<0.05$ ) entre el IMC y el Índice de Comedor Emocional (Tabla 4).

\section{Actividad física}

Respecto a la práctica de actividad física, el $54.7 \%$ consideró que aumentó la relación de actividad física durante el tiempo de confinamiento; el $27.5 \%$ se mantuvo igual; y el $17.8 \%$ reportó que disminuyó la realización de actividad física.

De acuerdo al Cuestionario de Actividad Física, se encontró lo siguiente: el $51.1 \%$ no practicaba deporte intenso (correr o jugar al fútbol) que implicara una aceleración importante en la respiración o el ritmo cardiaco durante al menos 10 minutos consecutivos, el $48.9 \%$ sí lo hacía.

La media de días a la semana en los que practicaban deporte intenso fue de 3.78 días (D.E.=1.54). El 43.7\% reportó que en esos días dedicaban de 30 a 60 minutos; el $28.4 \%$ de 60 a 90 minutos; el $13.6 \%$ menos de 30 minutos; el $11.1 \%$ de 90 a 120 minutos; y el $3.2 \%$ más de 90 minutos.

Respecto a los minutos a la semana que dedica el alumnado a la práctica de actividad física intensa, el 36.5\% no alcanzaba los minutos recomendados por la OMS (2020), ya que acumulaban menos de 75 minutos a la semana; mientras que el $63.5 \%$ sí cumplía con lo recomendado.

Respecto a la actividad física de intensidad moderada (montar en bicicleta, caminar de prisa, etc.), el $48.7 \%$ sí la realizaba y el $51.3 \%$ no lo hacía. La medía de días que dedicaban a la práctica de este tipo de actividad fue de 3.48 días (D.E.= 1.47).

El tiempo dedicado a la actividad física moderada fue: el $46.6 \%$ de 30 a 60 minutos; el $29.8 \%$ menos de 30 minutos; el $13.5 \%$ de 60 a 90 minutos; el $7 \%$ de 90 a 120 minutos; y el $3.1 \%$ más de 120 minutos.

De acuerdo al cálculo de minutos a la semana, se encontró que el $85.6 \%$ no alcanzaba la cantidad mínima recomendad por la OMS (2020) para actividad física de intensidad moderada, mientras que el $14.4 \%$ sí realiza 150 minutos o más a la semana de este tipo de actividades.

En torno al tiempo realizando actividades sedentarias, el $41.6 \%$ pasaba más de 120 minutos; el $17.1 \%$ de 30 a 60 minutos; el $14.4 \%$ de 60 a 90 minutos; el $14.2 \%$ menos de 30 minutos; y el $12.7 \%$ de 60 a 90 minutos. 
Tabla 3. Media y Desviación Estándar de la Escala de Afecto Positivo y Negativo PANAS-20.

\begin{tabular}{|l|c|c|}
\hline \multicolumn{1}{|c|}{ Ítems } & $\overline{\mathbf{X}}$ & D.E \\
\hline Estados afectivos positivos & & 1.04 \\
\hline Interesado/a & 2.37 & .963 \\
\hline Estimulado/a & 2.19 & 1.04 \\
\hline Enérgico/a & 2.46 & 1.12 \\
\hline Entusiasmado/a & 2.77 & 1.15 \\
\hline Orgulloso/a & 2.79 & 1.12 \\
\hline Alerta & 2.41 & 1.19 \\
\hline Inspirado/a & 2.67 & 1.13 \\
\hline Decidido/a & 2.83 & 1.13 \\
\hline Atento/a & 2.92 & 1.17 \\
\hline Activo/a & 2.89 & 1.05 \\
\hline Estados afectivos negativos & & .96 \\
\hline Tenso/a & 2.37 & .98 \\
\hline Disgustado/a & 2.05 & .95 \\
\hline Culpable & 1.72 & .82 \\
\hline Asustado/a & 1.72 & 1.07 \\
\hline Hostil & 1.63 & 1.00 \\
\hline Irritable & 2.06 & 1.14 \\
\hline Avergonzado/a & 1.72 & 1.05 \\
\hline Nervioso/a & 2.25 & .95 \\
\hline Miedoso/a & 1.89 & 1.67 \\
\hline Atemorizado/a & & \\
\hline
\end{tabular}

Nota: Las opciones de respuesta fueron: 1= "Nada", 2= "Un poco", 3= "Bastante", 4= "Mucho", 5= "Muchísimo".

Tabla 4. Análisis correlacional de Spearman de índice de Comedor Emocional y estados afectivos.

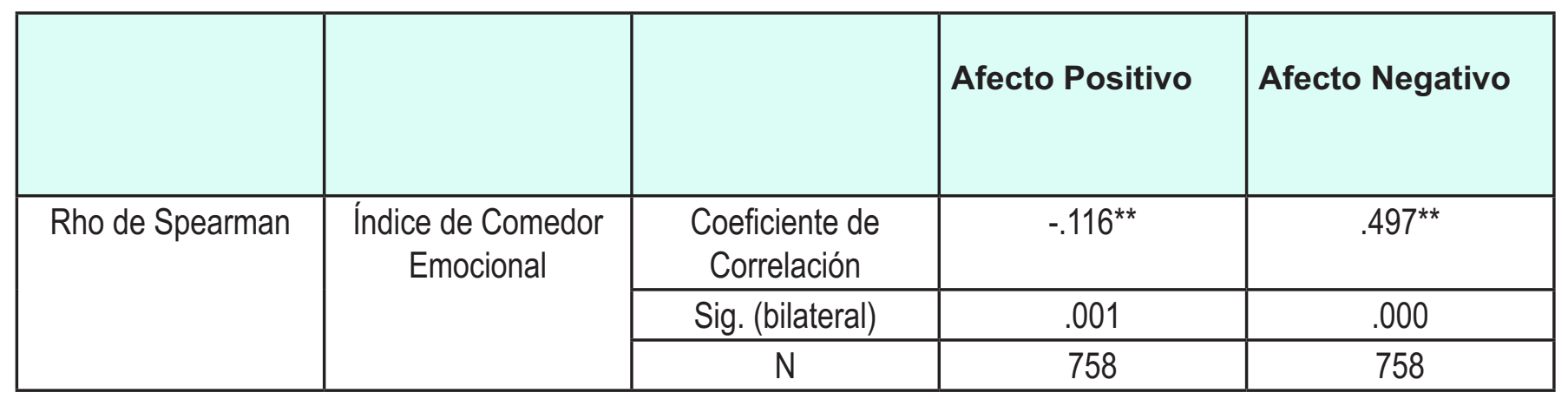

** La correlación es significativa en el nivel 0.01 (bilateral). 


\section{Discusión}

La enfermedad por la COVID-19 y sus riesgos ampliamente difundidos remarcan que nos encontramos frente a un fenómeno histórico de salud que ha traído grandes cambios en las dinámicas cotidianas con sus respectivas consecuencias sociales, económicas y sanitarias que prevalecerán a corto, mediano y largo plazo (Del-Río et al., 2020).

Dentro de las particularidades de la población universitaria a nivel de licenciatura se identifican ciertos factores de protección como los recursos económicos, sociales y emocionales disponibles para asimilar la información referente a la situación de pandemia (CortésMeda \& Ponciano-Rodríguez, 2021). Estos factores favorecen el proceso de adaptación a la nueva rutina postpandémica caracterizada por la virtualidad y la modificación de hábitos.

La presente investigación tuvo como primer objetivo analizar los hábitos de actividad física y alimentación. Al respecto, se encontró que los universitarios consideraron que la práctica de actividad física aumentó durante el tiempo de confinamiento. Estos hallazgos contradicen en parte lo encontrado por Ruiz-Zaldibar et al. (2021), quien reportó que en hombres la actividad física había disminuido significativamente y que en las mujeres aumentó. No obstante, es importante recalcar que el $41.6 \%$ de los universitarios analizados reportaron que permanecían más de 120 minutos al día realizando actividades sedentarias, las cuales se asocian a sus actividades académicas como asistir a clase en línea, realizar tareas académicas y mantener una comunicación con sus compañeros a través de medios digitales, lo cual referiría a que las actividades sedentarias también han incrementado durante la pandemia (Ammar et al., 2020), y al mismo tiempo, haber aumentado el tiempo disponible para realizar actividad física.

A pesar de lo reportado en el párrafo anterior, los resultados referentes al incremento en la práctica de actividad física y en las actividades sedentarias, también han sido reportados en el estudio de Romero-Blanco et al. (2020), lo que refiere a una mayor disponibilidad de tiempo, y que se puede relacionar a lo encontrado en la investigación de Salgado (2018), en la que se encontró que los universitarios preferían hacer actividad física solos en casa.

Por otro lado, la mayoría de los universitarios ha cambiado su alimentación durante la pandemia. La mayoría aumentó la ingesta de alimentos, resultado similar encontrado por Ruiz-Zaldibar et al. (2021) y por Osorio y Prado (2021) en población universitaria, quienes reportaron que el seguimiento de una dieta equilibrada y el cuidado de peso empeoró durante la pandemia.

Similar al estudio realizado por Salgado (2018), la población universitaria no se ve totalmente implicada en la preparación de alimentos, siendo el promedio de participación un $56.53 \%$ y la madre la principal encargada del proceso de preparación. La similitud con resultados de investigaciones previas pre-pandemia evidencian que los comportamientos asociados a la alimentación no han cambiado en cuanto a la implicación en los procesos, lo que se encontró también en la investigación de Dragun et al. (2021), estableciendo además como una ventaja del confinamiento, el ingerir los alimentos sin la presión de tener que salir de casa para asistir a clase.

Respecto a la forma de preparación de los alimentos, en nuestro estudio se pudo observar que la mayor parte de la población universitaria tiene una preferencia por elaborar alimentos de manera guisada o estofada (56.53\%), resultado similar a lo encontrado en la investigación de Gallardo (2021) y Zurita, Di Prieto y Robalino (2019). Esta técnica de preparación se caracteriza por la manera en la que los alimentos liberan sus nutrientes y minerales a través de la inclusión de agua o caldo (Nieto, 2014; Valdéz, 2020; Vilchez, 2020), lo que la convierte en una forma de preparación saludable y tradicional en la gastronomía mexicana.

Sin embargo, y tomando como guía el segundo objetivo propuesto en la presente investigación en relación a la relación entre comportamiento alimentario y estados emocionales, la alimentación también se ve influenciada por los estados afectivos que experimentan los universitarios. Hemos identificado que las medias más altas dentro del Cuestionario del Comedor Emocional se relacionaron con la presencia de antojos y emociones afines a la pandemia como el estrés, el enfado o el aburrimiento.

La relación entre el comportamiento alimentario y el estrés ha sido documentado en la investigación de Palomino-Pérez (2020), quien demostró que el estrés percibido, agudo o crónico, se asocia a un pobre autocontrol, lo cual favorece el consumo de alimentos poco saludables y con ello una alta ingesta emocional (Martínez, Bernal-Gómez, Mora, \& Hun, 2020). El estrés es capaz de modificar la forma de interacción en el entorno en el que nos desenvolvemos y en el comportamiento alimentario, al estar influenciado por las emociones (Cortés et al., 2018).

Al respecto, es relevante hacer mención de las diferencias identificadas entre hombres y mujeres y el Índice de Comedor Emocional, en el que se encontró que las universitarias presentaban un índice más alto, lo que 
coincide con lo reportado por Palomino-Pérez (2020), quien además explica que la ingesta de alimentos altos en azúcares y grasa se vuelven un medio para afrontar emociones negativas, y que se refuerza mediante los efectos que se presentan a nivel de amígdala, lo que favorece el estado de ánimo y la sensación de bienestar asociados al incremento en la serotonina.

Los efectos psicológicos de la pandemia se han generado, en gran medida, por factores diversos como la desinformación, la sobre-exposición al evento traumático, etc., que han incrementado los niveles de estrés en la población (Apaza, Seminario, \& Santa-Cruz, 2020). A pesar de lo anterior, en la presente investigación el alumnado universitario manifestó sentirse atento y estimulado con mayor frecuencia. Sin embargo, al contrastar con los estados afectivos negativos, se encontró que las emociones más recurrentes fueron sentirse tenso y nervioso, lo que coincide con lo reportado por GonzalezRamirez et al. (2020), y que se puede atribuir a la falta de cercanía generada por el distanciamiento social, que también coincide con lo reportado por Ruiz-Zaldibar et al. (2021), que encontró que la relación con la familia y amigos había empeorado durante la pandemia.

La población universitaria se encuentra en un período crítico para la adopción y mantenimiento de estilos de vida saludables, la situación actual de pandemia ha mermado sus dinámicas cotidianas, lo que implica una importante necesidad de promover estilos de vida saludables. Cabe resaltar que si bien, el índice de Comedor Emocional presentó una correlación positiva moderada con los estados afectivos negativos, los universitarios presentaron emociones positivas con mayor frecuencia. Igualmente, otro importante factor de protección que se ha visto incrementado es la práctica de actividad física.

\section{Conclusiones}

La situación suscitada por la pandemia por la COVID-19 ha tenido un impacto negativo considerable en cuestiones académicas, de salud y de estilo de vida (Son et al., 2020), que han implicado también un importante riesgo para la salud mental, independientemente del grupo de edad al que se pertenezca (Giuntella et al., 2021).

Tal como lo plantearon Salgado-Espinosa y Álvarez (2020), el estado de salud de la población universitaria se construye por la sinergia de los hábitos de vida saludable y los recursos con los que cuenten, que se pueden ver incrementados o disminuidos por la situación histórica que atraviesan y el contexto cultura en el que se desarrollan.

El estudio de las implicaciones en estado de salud y estados emocionales de alumnado universitario permitirá generar estrategias que les oriente en la toma de decisiones adecuadas para la adopción y mantenimiento de comportamientos que favorezcan el estado de su salud (Cortés-Meda \& Ponciano-Rodríguez, 2021), abarcando la esfera biopsicosocial.

En este sentido, cabe resaltar que a pesar de la situación el alumnado incrementó su práctica de actividad física y las emociones más frecuentemente experimentadas correspondieron a estados afectivos positivos. Sin embargo, su alimentación se ha visto afectada al consumir más alimentos o menos que antes de la pandemia, aunque sería relevante que, para futuras investigaciones, se profundice en cómo era su alimentación pre-pandemia, ya que el incremento o disminución de la alimentación no necesariamente implicaría un mayor riesgo de malnutrición, si sus hábitos previos no eran los adecuados.

Dentro de las limitaciones de la investigación, se encuentra que, por el tipo de muestreo utilizado (no probabilístico por conveniencia), no es posible generalizar los resultados. Sería interesante realizar investigaciones en otros contextos culturales o incluso en otros niveles educativos para identificar cuál ha sido el impacto en los hábitos de salud de los estudiantes y los estados afectivos experimentados.

\section{Contribuciones de los autores}

Los autores participaron igualmente en la elaboración del manuscrito y aprobaron la versión final presentada.

\section{Financiación}

\section{Esta investigación no recibió financiación.}

\section{Declaración de disponibilidad de datos}

Los datos presentados en este estudio pueden ser solicitados al autor de correspondencia.

\section{Agradecimientos}

Agradecemos al director general de Universidad Vizcaya de las Américas campus Saltillo, el licenciado Emmanuel Berumen Aguirre por su apoyo para la realización de este estudio, así como a los coordinadores y alumnado de las diferentes titulaciones que participaron en la presente investigación.

\section{Conflicto de interés \\ Los autores declaran que no hay conflicto de interés.}

\section{Referencias bibliográficas}

Del-Río, C., Alcocer-Gamba, M.A., Escudero-Salamanca, M., Galindo-Fraga, A., Guarner, J., \& Escudero, X. (2020). La pandemia de coronavirus SARS CoV-2 
(COVID-19): situación actual e implicaciones para México. Cardiovascular and Metabolic Science, 31(S3), 170-177. https://doi.org/10.35366/93943

Aburto-Morales, J. S., Romero-Méndez, J., LucioGarcía, C. A., \& Madrigal-Bustamante, J. A. (2020). México ante la epidemia de COVID-19 (SARSCoV-2) y las recomendaciones al Subsistema Nacional de Donación y Trasplante. Revista Mexicana de Trasplantes, 9(1), 6-14. https://doi. org/10.35366/94024

Achón, M., González, M. P., \& Varela, G. (2018). Criterios de armonía funcional entre gastronomía y salud: una visión desde la comunidad científica. Nutricion Hospitalaria, 35(4), 75-84. https://doi.org/10.20960/ nh.2131

Álvarez-Bermudez, J., \& Meza-Peña, C. (2021). Las repercusiones de la pandemia de Covid-19 en los hábitos de salud y la adaptación a la misma: caso México. Salud Pública de México, 63(4), 2021. https://doi.org/https://doi.org/10.21149/12626

Amatori, S., Zeppa, S. D., Preti, A., Gervasi, M., Gobbi, E., Ferrini, F., ... Sisti, D. (2020). Dietary habits and psychological states during covid-19 home isolation in italian college students: The role of physical exercise. Nutrients, 12(12), 1-17. https://doi.org/10.3390/ nu12123660

Ammar, A., Brach, M., Trabelsi, K., Chtourou, H., Boukhris, O., Masmoudi, L., ... Hammouda, O. (2020). Effects of COVID-19 Home Confinement on Eating Behaviour and Physical Activity: Results of the ECLB-COVID19 International Online Survey. Nutrients, 12(1583), 13. https://doi.org/https://doi.org/10.3390/nu12061583

Annesi, J. J. (2012). Supported exercise improves controlled eating and weight through its effects on psychosocial factors: extending a systematic research program toward treatment development. The Permanente Journal, 16(1), 7-18. https://doi. org/10.7812/11-136

Apaza, C. M., Seminario, R. S., \& Santa-Cruz, J. E. (2020). Factores psicosociales durante el confinamiento por el Covid-19- Perú. Revista Venezolana de Gerencia, 25(90), 402-413. https://www.redalyc. org/journal/290/29063559022/html/

Barrera, J., Bicenty, A., Gerena, R., Ibañez, É., Martínez, J., \& Thomas, Y. (2008). Cambios de hábitos alimentarios de los estudiantes de odontología de la Fundación
Universitaria San Martín de Bogotá, Colombia. Nova, 6(9), 27. https://doi.org/10.22490/24629448.393

Bravo-Cucci, S., Cruz-Gonzales, G., Medina-Espinoza, R., \& López-Guevara, N. (2020). Actividad física en estudiantes universitarios beneficiarios de un programa nacional de becas de inclusión social, Perú, 2016. Revista del Grupo de Investigación en Comunidad y Salud, 5(2), 78-97.

Catalán-Matamoros, D. (2020). La comunicación sobre la pandemia del COVID-19 en la era digital: manipulación informativa, fake news y redes sociales. Revista Española de Comunicación en Salud, Supl. 1, S5-S8. https://doi.org/10.20318/recs.2020.5531

Celorio-Sardà, R., Comas-Basté, O., Latorre-Moratalla, M. L., Zerón-Rugerio, M. F., Urpi-Sarda, M., IllánVillanueva, M., ... Vidal-Carou, M. D. C. (2021). Effect of covid-19 lockdown on dietary habits and lifestyle of food science students and professionals from Spain. Nutrients, 13(5), 1-13. https://doi.org/10.3390/ nu13051494

Chowdhury, A. I. (2020). Role and Effects of Micronutrients Supplementation in Immune System and Role and Effects of Micronutrients Supplementation in Immune System and SARS-Cov-2 ( COVID-19). Asian Journal of Immunology, 4(2), 47-55.

Cortés-Meda, A., \& Ponciano-Rodríguez, G. (2021). Impacto de los determinantes sociales de la COVID-19 en México. Boletín Sobre COVID-19, UNAM., 2(17), 9-13.

Cortés, C. E., Escobar, A., Cebada, J., Soto, G., Bilbao, T., \& Vélez, M. (2018). Estrés y cortisol: implicaciones en la ingesta de alimento. Revista Cubana de Investigaciones Biomédicas, 37(3), 1-15. http:/l scielo.sld.cu/scielo.php?script=sci_arttext\&pid =S0864-03002018000300013

Dragun, R., Veček, N. N., Marendić, M., Pribisalić, A., Đivić, G., Cena, H., ... Kolčić, I. (2021). Have lifestyle habits and psychological well-being changed among adolescents and medical students due to COVID-19 lockdown in Croatia? Nutrients, 13(1), 1-16. https:// doi.org/10.3390/nu13010097

Fajardo, E., Camargo, Y., Buitrago, E., Peña, L., \& Rodríguez, L. (2016). Estado nutricional y preferencias alimentarias de una población de estudiantes universitarios en Bogotá. Revista Med, 24(2), 58-65. https://doi.org/10.18359/rmed.2641 
Gallardo, E. P. (2021). Hábitos alimentarios en estudiantes de la Universidad Técnica del Norte en tiempos de COVID-19, año 2021. Universidad Técnica del Norte.

Garaulet, M., Canteras, M., Morales, E., López-Guimera, G., Sánchez-Carracedo, D., \& Corbalán-Tutau, M. D. (2012). Validation of a questionnaire on emotional eating for use in cases of obesity; the Emotional Eater Questionnaire (EEQ). Nutricion Hospitalaria, 27(2), 645-651. https://doi.org/10.3305/nh.2012.27.2.5659

Giuntella, O., Hyde, K., Saccardo, S., \& Sadoff, S. (2021). Lifestyle and mental health disruptions during COVID-19. Proceedings of the National Academy of Sciences of the United States of America, 118(9). https://doi.org/10.1073/pnas.2016632118

Gonzalez-Ramirez, J., Mulqueen, K., Silverstein, S., Mulqueen, C., \& BuShell, S. (2020). Emergency Online Learning: College Students' Perceptions during the COVID-19 pandemic. College Student Journal, 55(1), 1-34.

González, M. (2018). Cuestionario de Comedor Emocional (CCE) en Chile. Gen, 72(1). http:// ve.scielo.org/scielo.php?script=sci_arttext\&pid $=$ S0016-35032018000100005

Johnson, M. C., Saletti-Cuesta, L., \& Tumas, N. (2020). Emotions, concerns and reflections regarding the COVID-19 pandemic in argentina. Ciencia \& Saude Coletiva, 25, 2447-2456. https://doi. org/10.1590/1413-81232020256.1.10472020

López, B. A., Soberanes, A., \& Loaiza, A. (2021). Aplicación de métodos de cocción saludables. Boletín Científico de Las Ciencias Económico Administrativas Del ICEA, 9(18), 55-56. https:// doi.org/https://doi.org/10.29057/icea.v9i18.7068

Mantilla, S. C., \& Gómez-Conesa, A. (2007). El Cuestionario Internacional de Actividad Física. Un instrumentoadecuadoen el seguimiento delaactividad física poblacional. Revista Iberoamericana de Fisioterapia y Kinesiologia, 10(1), 48-52. https:// doi.org/10.1016/S1138-6045(07)73665-1

Martínez, T. Y., Bernal-Gómez, S. J., Mora, A. P., \& Hun, N. E. (2020). Percepción subjetiva de manejo emocional, ansiedad y patrones de ingesta relacionados con aislamiento por COVID-19. Universitas Psychologica, 19, 1-9. https://doi. org/10.11144/Javeriana.upsy19.epbi
Moral, J. (2011). La escala de afecto positivo y negativo (PANAS) en parejas casadas mexicanas. CIENCIA Ergo Sum, 18, 117-125.

Nieto, C. (2014). Técnicas de cocción: sabor, color, textura y nutrientes a buen recaudo. Farmacia Profesional, 28(3), 15-19. https://www.elsevier.es/es-revistafarmacia-profesional-3-pdf-X0213932414396318

Noguera, F., Gigante, S., Menoni, C., Aude, I., Montero, D., \& Peña, N. (2018). Principios de la preparación de alimentos. Universidad de la República de Uruguay.

Organización Mundial de la Salud. (2014). Cuestionario Mundial sobre Actividad Física. Departamento de Enfermedades Crónicas y Promoción de La Salud, 3. www.who.int/chp/steps\%0Ahttps://www.who.int/ ncds/surveillance/steps/GPAQ_ES.pdf

Organización Mundial de la Salud. (2020). Actividad física. https://www.who.int/es/news-room/factsheets/detail/physical-activity

Osorio, M., \& Prado, C. (2021). Efectos económicos, escolares y de salud del COVID-19 en una muestra de estudiantes universitarios mexicanos. Enseñanza e Investigación en Psicología, 3(1), 90-100. https:// revistacneip.org/index.php/cneip/article/view/178/113

Palomino-Pérez, A. M. (2020). Rol de la emoción en la conducta alimentaria. Revista Chilena de Nutrición, 47(2), 286-291. https://doi.org/10.4067/ s0717-75182020000200286

Peña-y-Lillo, M. (2019). Utilidad de la Teoría de la Conducta Planificada para entender el consumo de frutas y verduras: evidencia de estudios en adultos y adolescentes chilenos. Revista Española de Comunicación en Salud, 10(1), 50-61.

Peña, E., \& Reidl, L. M. (2015). Las Emociones y la Conducta Alimentaria. Acta de Investigación Psicológica, 5(3), 2182-2193. https://doi. org/10.1016/s2007-4719(16)30008-4

Pérez, S. E., Vega-García, L. A., \& Romero-Juárez, G. (2007). Prácticas alimentarias de mujeres rurales: ¿Una nueva percepción del cuerpo? Salud Pública de México, 49(1), 52-62. https://doi.org/10.1590/ S0036-36342007000100008

Reyes, S., \& Canto, M. O. (2020). Conocimientos sobre alimentación saludable en estudiantes de una universidad pública. Revista Chilena de Nutrición, 
47(1), 67-72. https://doi.org/10.4067/s071775182020000100067

Robles, R., \& Páez, F. (2003). Estudio sobre la traducción al Español y las propiedades psicométricas de las escalas de Afecto Positivo y Negativo (PANAS). Salud Mental, 26(1), 69-75.

Rojas-Vichique, J. A., Quintero-Pereda, S., \& CarmonaFigueroa, Y. P. (2020). Alimentación emocional durante tiempos de COVID-19 en adultos jóvenes de 18 a 29 años . Revista Mexicana de Medicina Forense, 5, 66-71. https://revmedforense.uv.mx/ index.php/RevINMEFO/article/view/2906/4802

Rojas, A. T., \& García-Méndez, M. (2017). Construcción de una Escala de Alimentación Emocional. Revista Iberoamericana de Diagnóstico y Evaluación-e Avaliação Psicológica, 3(45), 85-95. https://doi. org/10.21865/RIDEP45.3.07

Romero-Blanco, C., Rodríguez-Almagro, J., OnievaZafra, M. D., Parra-Fernández, M. L., Prado-Laguna, M. D. C., \& Hernández-Martínez, A. (2020). Physical activity and sedentary lifestyle in university students: Changes during confinement due to the covid-19 pandemic. International Journal of Environmental Research and Public Health, 17(18), 1-13. https:// doi.org/10.3390/ijerph17186567

Romero, N. (2018). Afecto, alexitimia e ingesta emocional en personas con sobrepeso y obesidad [Universidad Autónoma de Nuevo León]. http://dspace.unitru.edu.pe/handle/UNITRU/10525

Ruiz-López, L., Felipe-Ortega-Fonseca, X., VázquezCuriel, R. A., \& Balderrama-Carmona, A. P. (2021). Evaluación nutricional en universitarios y recomendación de una dieta óptima para fortalecer el sistema inmunitario contra la COVID-19. Horizonte Sanitario, 20(3), 417-425. https://doi.org/10.19136/ hs.a20n3.4036

Ruiz-Zaldibar, C., García-Garcés, L., Vicario-Merino, A., Mayoral-Gonzalo, N., Lluesma-Vidal, M.,... PérezManchón, D. (2021). University student's lifestyle changes during COVID-19 lockdown: A Spanish Cross-Sectional Survey. Research Square, 1-20. https://doi.org/https://doi.org/10.21203/ rs.3.rs-195049/v1

Sainz, P., Ferrer, M. C., \& Sánchez, E. (2016). Cooking Skills and Consumption of Ready Meal in University
Students of Barcelona, Spain. Revista Española de Salud Pública, 90, e1-e13.

Salgado-Espinosa, M. L., \& Álvarez, J. (2020). Autoeficacia: Relación con los hábitos de salud y la calidad de vida en universitarios del noreste de México. Revista Española de Comunicación en Salud, 11(1), 53-64. https://doi.org/10.20318/ recs.2020.5166

Salgado, M. L. (2018). Relación de los hábitos de salud, la imagen corporal y la autoeficacia con la calidad de vida de estudiantes universitarios del noreste de México. In Undefined. Universidad Autónoma de Nuevo León.

Sánchez Benito, J. L., \& Pontes Torrado, Y. (2012). Influencia de las emociones en la ingesta y control de peso. Nutricion Hospitalaria, 27(6), 2148-2150. https://doi.org/10.3305/nh.2012.27.6.6061

Sedó, P., Chinnock MC, A., \& Piedra, M. I. (2000). Propuesta metodológica para el mejoramiento nutricional de preparaciones comunes en la dieta. Revista Costarricense de Salud Pública, 9(16).

Son, C., Hegde, S., Smith, A., Wang, X., \& Sasangohar, F. (2020). Effects of COVID-19 on college students' mental health in the United States: Interview survey study. Journal of Medical Internet Research, 22(9). https://doi.org/10.2196/21279

Tazeoğlu, A., Bozdoğan, F., \& İdiz, C. (2021). Evaluation of Osmaniye Korkut Ata university students' eating behaviors during the quarantine period during the COVID-19 pandemic period. Nutrición Clínica y Dietética Hospitalaria, 41(2), 86-93. https://doi. org/10.12873/412tazeoglu

Valdéz, J. M. (2020). Proyecto para la mejora de los conocimientos y hábitos de alimentación a través de la implmentación de una guía de alimentación saludable basada en un presupuesto mensual y dirigida al personal de Traumaservice Internacional S.A. de C.V. [Universidad de Guadalajara]. https:// riudg.udg. $m x / v i s o r / p d f j s / v i e w e r . j s p ? i n=j \& p$ $\mathrm{df}=20.500 .12104 / 81776 / 1 / \mathrm{MCUCS} 10035 \mathrm{FT}$.pdf

Varela, G. (2016). Gastronomía, comunicación y salud. Influencia de la gastronomía en los hábitos alimentarios de la población. Revista Española de Comunicación en Salud, 7(Sup1), 85-94. https:// doi.org/10.20318/recs.2016.3125 
Vilchez, V. (2020). Influencia del conocimiento de los cuidadores en alimentación y nutrición en el estado nutricional de preescolres de una I.E.I. en el Distrito de Santa Anita, 2018 [Universidad Científica del Sur]. https://repositorio.cientifica.edu. pe/handle/20.500.12805/1723

Watson, D., Clark, L.A., \& Tellegen,A. (1988). Development and validation of brief measures of positive and negative affect: the PANAS scales. Journal of Personality and Social Psychology, 54(6), 10631070. https://doi.org/10.4135/9781483398839.n13

Zurita, R., Di Prieto, M. T., \& Robalino, J. (2019). Alimentación saludable y uso de técnicas culinarias. La Ciencia al Servicio de la Salud y la Nutrición, 10, 270-280. http://revistas.espoch.edu.ec/index. php/cssn/article/view/270 